Scientific Journal Warsaw University of Life Sciences - SGGW

Problems of World Agriculture volume 17 (XXXII), number 4, 2017: 199-209

DOI: $10.22630 /$ PRS.2017.17.4.96

Justyna Lapińska, ${ }^{1}$ Ewa Zdunek-Rosa ${ }^{2}$, Maurycy Graszewicz ${ }^{3}$

Nicolaus Copernicus University in Toruń

\title{
Poland's Competitive Position in Trade in Agri-Food Products with the United Kingdom - Selected Aspects
}

\begin{abstract}
Thirteen years have passed since Poland's accession to the European Union. This is a period long enough to make some generalisations over the accession results. This paper focuses on assessing the competitive position of Poland in trade in agri-food products with one of its most important trading partners - the United Kingdom. For this purpose, quantitative indexes of competitive positioning were used, in particular, the trade coverage ratio as well as the revealed comparative advantage indexes - RCAi and LFIi. The conducted analyses show that Poland's competitive position in agri-food trade with the United Kingdom improved markedly over the period considered. The dynamic growth in trade, especially in exports, a significant increase in the trade balance surplus, and generally favourable comparative advantage indexes for Poland show that the period of EU membership has been well utilised by Polish food producers. Polish food is increasingly eagerly bought by demanding British consumers.
\end{abstract}

Key words: competitive position, agri-food products, foreign trade

JEL Classification: Q17, F14

\section{Introduction}

Accession to the European Union meant for Poland the abolition of restrictions in trade exchange, including on the agri-food market. When opening its domestic market, Poland was granted the opportunity to sell its products on the developed European market. After thirteen years of Poland functioning in the EU structures, it can be stated that EU membership has proved to be very beneficial for the Polish food industry, and the results of Poland's foreign trade in agri-food products confirm this. Polish agri-food products are well known and eagerly acquired by European consumers. This is primarily due to the use of modern processing technologies, high quality raw materials, and price advantages (Szczepaniak, 2011).

The purpose of this paper is to analyse and evaluate the competitive position of Polish agri-food products, after Poland's accession to the European Union, in foreign trade exchange with one of the most important trading partners - the United Kingdom. The UK is an important trading partner for Poland in the European Union and is preparing to leave the Community. Its departure will undoubtedly influence the development of future economic relations with the remaining EU countries. It is, therefore, a very good time to analyse and summarize Poland's thirteen-year trade cooperation with the United Kingdom within the EU.

\footnotetext{
${ }^{1}$ Doctor habilitated, professor of the NCU, ul. Gagarina 13A, 87-100 Toruń, e-mail: justlap@umk.pl

${ }^{2}$ PhD, e-mail: ezdunek@umk.pl

${ }^{3} \mathrm{PhD}$, e-mail: mgraszewicz1@swps.edu.pl
} 


\section{Materials and methods}

The subject literature broadly presents and discusses the results that Poland has achieved in agri-food trade. Authors of various analyses and reports (see, for example, Ambroziak, 2013, 2016; Pawlak, 2014; Szczepaniak, 2015) agree that membership in the EU has become a very strong incentive for Poland to develop trade with EU countries and that Polish food producers have significantly improved their competitive position in the enlarged European Union market. Poland's integration with the EU has initiated the modernization of Polish agri-food companies, also through the inflow of foreign direct investment and modern technologies (Firlej, 2010; Łapińska, 2014).

In general, the subject literature divides a country's measures and methods of assessing the competitive position into two categories. The first refers to the efficiency of a country's functioning in the international exchange of goods and services (measures and methods of assessing the ability to sell). The other includes measures and methods of assessing the attractiveness of a country's mobile production factors (the ability to attract) and of maintaining international technological competitiveness (Misala, 2007).

This paper focuses on evaluating the competitive position of Poland by means of selected measures belonging to the first group, which were applied to agri-food trade exchange with the United Kingdom. The assessment of the competitive position was preceded by a brief presentation of the results of foreign trade in this type of products with the United Kingdom.

The study covered the goods belonging to the SITC 0 , SITC 1 and SITC $4^{4}$ sections for the years 2004-2016. These three sections cover all agri-food products. The analyses conducted were based on statistical data published by Eurostat ${ }^{5}$.

To assess the competitive position of countries participating in international trade, analysis of the level of revealed comparative advantages is often applied. The output of world science in the field of the methods of calculating comparative advantages is significant (see, for example, Balassa, 1965; Vollrath, 1991; Hoen, Oosterhaven, 2006). In this work, two mutually complementary indexes were used to evaluate the comparative advantages. The first of these is the $R C A_{i}$ index, constructed in accordance with the GruppLegler formula (Gehrke Grupp, 1994):

$$
\mathrm{RCA}_{\mathrm{i}}=\ln \left(\frac{\mathrm{x}_{\mathrm{i}}}{\mathrm{m}_{\mathrm{i}}}: \frac{\mathrm{x}}{\mathrm{M}}\right)
$$

where:

$x_{i}$ - exports of product (group of products) $i$,

$m_{i}$-imports of product (group of products) $i$,

$X$ - country's total exports,

\footnotetext{
${ }^{4}$ Standard International Trade Classification (SITC) - a classification, which is used to provide aggregated data. Aggregated data on trade are often presented in the one- or two- and three-digit categories of the SITC. An example of a one-digit category (section) is SITC 1 'food and live animals' and of a two-digit category (divisions) is 01 'meat and meat preparations' and three-digit category (groups) SITC 022 'milk and cream'.

SITC Rev. 4 was accepted by the United Nations Statistical Commission. It comprises 2970 basing headings which are amalgamated into 262 groups, 67 divisions and 10 sections.

${ }^{5}$ Eurostat Database, http://ec.europa.eu/eurostat/data/database.
} 
$M$ - country's total imports.

The other index is $L F I_{i}$ - Lafay's formula (1992) modified by Bugamelli (2001):

$$
\mathrm{LFI}_{\mathrm{i}}=100 \times\left(\frac{\mathrm{X}_{\mathrm{i}}-\mathrm{M}_{\mathrm{i}}}{\mathrm{X}_{\mathrm{i}}+\mathrm{M}_{\mathrm{i}}}-\frac{\sum_{\mathrm{i}=1}^{\mathrm{N}}\left(\mathrm{X}_{\mathrm{i}}-\mathrm{M}_{\mathrm{i}}\right)}{\sum_{\mathrm{i}=1}^{\mathrm{N}}\left(\mathrm{X}_{\mathrm{i}}+\mathrm{M}_{\mathrm{i}}\right)}\right) \times \frac{\mathrm{X}_{\mathrm{i}}+\mathrm{M}_{\mathrm{i}}}{\sum_{\mathrm{i}=1}^{\mathrm{N}}\left(\mathrm{X}_{\mathrm{i}}+\mathrm{M}_{\mathrm{i}}\right)},
$$

where:

$X_{i}$ - exports of product (group of products) $i$,

$M_{i}$ - imports of product (group of products) $i$.

Both indicators are interpreted in the same way. A value greater than zero means the occurrence of a revealed comparative advantage. It also indicates its intensity. A negative value of the indicator means no comparative advantage.

The revealed comparative advantages were designated for product groups according to the SITC nomenclature. The first of the indexes $\left(R C A_{i}\right)$ was used to assess the competitiveness of agri-food products in total trade volume with the United Kingdom. The second indicator $\left(L F I_{i}\right)$ was used to assess the competitiveness of individual products (SITC three-digit groups) by examining it only against trade in agri-food products (also with the United Kingdom only). In the second case, it was assumed that $\sum_{i=1}^{N}\left(X_{i}+M_{i}\right)$ and $\sum_{i=1}^{N}\left(X_{i}-M_{i}\right)$ will only represent the turnover and balance in trade in agri-food products, and not Poland's total trade with the United Kingdom.

\section{Agri-food trade with the United Kingdom - selected aspects}

The United Kingdom is one of Poland's most important trading partners. In terms of its volume of exports, it is the second largest trading partner for Poland, with a $6.7 \%$ participation, following Germany which has $27.1 \%$. The United Kingdom is also one of the major sources of imports to the Polish market. Its share in Poland's imports is $2.7 \%^{6}$, (Rocznik Statystyczny Handlu Zagranicznego, 2016).

In the years 2004-2016 there was a significant increase in the Polish-British trade turnover. The total value of Poland's exports to the British market almost quadrupled, from 3.3 billion EUR to 12.1 billion EUR, while the value of imports from the United Kingdom almost doubled - from EUR 2.5 billion to EUR 5.1 billion.

Bilateral trading developed particularly well in the section that included machines, equipment and transport equipment. The turnover of these types of products accounted for almost 6.8 billion EUR (exports - 5.1 billion EUR, imports - 1.7 billion EUR), which constituted over $40 \%$ of Poland's total trade with the United Kingdom in 2016. Agri-food products were also an important commodity group in the Polish-British trade. In 2016, Polish exports of food products to the United Kingdom accounted for nearly 2.1 billion EUR, while imports were 0.5 billion EUR.

The share of these types of products in trade exchange between Poland and the United Kingdom significantly increased throughout the period considered. On the import side, the share of agricultural and food products increased by 7.6 percentage points in the years 2004-2016, reaching the level of $10.6 \%$. Regarding Poland's exports to the United

\footnotetext{
${ }^{6}$ The most important suppliers of goods to the Polish market include Germany with a $22.9 \%$ share, China -
} $11.6 \%$, Russia - 7.3\% (Rocznik Statystyczny Handlu Zagranicznego, 2016). 
Kingdom, the share of agricultural and food products increased in the same time period by 8.4 percentage points to $17.6 \%$ (see Table 1.)

Between 2004 and 2016 the value of Polish-British trade in agri-food products increased over seven-fold - from 372.5 million EUR to 2,665.5 million EUR. The dynamics of import and export growth was similar (see Table 1). Imports increased from 73.8 million EUR to 542.8 million EUR, while exports from EUR 298.7 million to EUR 2,122.7 million.

Table 1. Foreign trade between Poland and the United Kingdom in agri-food products in the years 2004-2016

\begin{tabular}{|c|c|c|c|c|c|c|c|c|c|c|c|c|c|}
\hline Specification & 2004 & 2005 & 2006 & 2007 & 2008 & 2009 & 2010 & 2011 & 2012 & 2013 & 2014 & 2015 & 2016 \\
\hline \multicolumn{14}{|c|}{ Imports } \\
\hline $\begin{array}{l}\text { Value } \\
\text { (in mln EUR) }\end{array}$ & 73.8 & 100.3 & 131.0 & 185.9 & 269.8 & 312.4 & 328.5 & 395.9 & 376.3 & 414.8 & 452.3 & 500.6 & 542.8 \\
\hline $\begin{array}{l}\text { Previous } \\
\text { year }=100\end{array}$ & 123.3 & 135.9 & 130.6 & 141.9 & 145.1 & 115.8 & 105.1 & 120.5 & 95.0 & 110.2 & 109.0 & 110.7 & 108.4 \\
\hline $\begin{array}{l}\text { Year } \\
2004=100\end{array}$ & 100.0 & 135.9 & 177.5 & 251.9 & 365.6 & 423.4 & 445.2 & 536.6 & 509.9 & 562.2 & 613.0 & 678.4 & 735.6 \\
\hline $\begin{array}{l}\text { Share of } \\
\text { agri-food } \\
\text { products in } \\
\text { total trade } \\
\text { (in } \% \text { ) }\end{array}$ & 3.0 & 3.9 & 4.3 & 5.1 & 6.8 & 9.3 & 8.2 & 9.5 & 9.5 & 9.6 & 9.9 & 9.7 & 10.6 \\
\hline \multicolumn{14}{|c|}{ Exports } \\
\hline $\begin{array}{l}\text { Value } \\
\text { (in mln EUR) }\end{array}$ & 298.7 & 397.8 & 540.8 & 742.9 & 813.9 & 790.3 & 935.5 & 1027.6 & 1322.9 & 1521.8 & 1676.7 & 2016.8 & 2122.7 \\
\hline $\begin{array}{l}\text { Previous } \\
\text { year }=100\end{array}$ & 167.5 & 133.2 & 135.9 & 137.4 & 109.5 & 97.1 & 118.4 & 109.8 & 128.7 & 115.0 & 110.2 & 120.3 & 105.3 \\
\hline $\begin{array}{l}\text { Year } \\
2004=100\end{array}$ & 100.0 & 133.2 & 181.0 & 248.7 & 272.4 & 264.5 & 313.1 & 344.0 & 442.8 & 509.4 & 561.2 & 675.1 & 710.5 \\
\hline $\begin{array}{l}\text { Share of } \\
\text { agri-food } \\
\text { products in } \\
\text { total trade } \\
\text { (in } \% \text { ) }\end{array}$ & 9.2 & 9.9 & 10.7 & 12.2 & 12.2 & 12.6 & 12.4 & 11.8 & 13.6 & 15.2 & 15.9 & 16.7 & 17.6 \\
\hline \multicolumn{14}{|c|}{ Balance } \\
\hline $\begin{array}{l}\text { Value } \\
\text { (in mln EUR) }\end{array}$ & 225.0 & 297.5 & 409.8 & 557.1 & 544.1 & 477.9 & 607.0 & 631.7 & 946.6 & 1107.0 & 1224.4 & 1516.2 & 1580.0 \\
\hline $\begin{array}{l}\text { The level of } \\
\text { covering the } \\
\text { import by } \\
\text { export (\%) }\end{array}$ & 404.9 & 396.6 & 412.8 & 399.7 & 301.7 & 252.9 & 284.8 & 259.5 & 351.6 & 366.9 & 370.7 & 402.9 & 391.1 \\
\hline
\end{tabular}

Source: Authors' own calculations based on Eurostat, http://ec.europa.eu/eurostat/data/database.

This bilateral trade turnover was growing markedly by the end of 2007. Poland's exports of food and beverage products to the United Kingdom increased by an annual average of $43.5 \%$, while imports to Poland by $32.9 \%$. From 2008, as a result of the economic slowdown triggered by the global financial crisis, the pace of growth of crossborder trade was decreasing. A significant drop in exports of Polish goods to the British 
market occurred in 2009. The value of Polish exports of agri-food products to the United Kingdom decreased by 23.6 million EUR compared to 2008.

In the following years, despite the initially difficult economic situation in the world, there was a revival of Polish-British trade in agri-food products. In the years 2011-2016 the value of Poland's exports to the United Kingdom was increasing by $14.8 \%$ annually, while import of British goods to the Polish market was rising by $9 \%$ annually.

\section{Evaluation of the competitive position of Poland's agri-food trade - analysis of selected indexes}

The most frequently used and at the same time the easiest indexes used for assessing the competitive position of a country in foreign markets are the development of trade balance volume and export-import relations in trade in particular goods or groups of goods. Over the period under review, Poland had a surplus in bilateral trade in agri-food products with the United Kingdom. In 2004 the positive balance amounted to EUR 225 million. In subsequent years, this positive balance in trade in agri-food products increased significantly. In 2016 Poland achieved a trade surplus of 1,580 million EUR in trade with the United Kingdom. The degree of coverage of imports by exports in the agri-food products trade was extremely favourable for Poland over the whole period considered. In the last year of the studied period, export revenue exceeded more than four times the value of imports.

Table 2. The revealed comparative advantage indexes RCAi in trade in agri-food products between Poland and the United Kingdom in the years 2004-2016 according to the SITC classification

\begin{tabular}{|c|c|c|c|c|c|c|c|c|c|c|c|c|c|c|}
\hline SITC & Specification & 2004 & 2005 & 2006 & 2007 & 2008 & 2009 & 2010 & 2011 & 2012 & 2013 & 2014 & 2015 & 2016 \\
\hline 001 & ive animals & 3 & -1.56 & -2.32 & 1.93 & 0.51 & 0.08 & .54 & -5.36 & -2.18 & .07 & -3.07 & -6.02 & -5.26 \\
\hline 011 & $\begin{array}{l}\text { Meat of bovine animals, } \\
\text { fresh, chilled or frozen }\end{array}$ & (.) & 4.73 & 3.46 & 3.19 & 2.78 & 1.80 & 0.42 & 0.54 & 1.08 & 1.42 & 1.18 & 1.62 & 1.61 \\
\hline 012 & $\begin{array}{l}\text { Meat and edible meat } \\
\text { offal, fresh, chilled or } \\
\text { frozen (except meat of } \\
\text { bovine animals) }\end{array}$ & 1.10 & 0.75 & 1.03 & 0.35 & -0.40 & -0.50 & -0.40 & -0.32 & -0.39 & -0.28 & -0.09 & 0.17 & 0.27 \\
\hline 016 & $\begin{array}{l}\text { Meat and edible meat } \\
\text { offal salted, dried or } \\
\text { smoked }\end{array}$ & -0.08 & 4.29 & (.) & (.) & 4.45 & 4.49 & 6.88 & 5.66 & 5.69 & 6.80 & 6.92 & 7.83 & 6.27 \\
\hline 017 & $\begin{array}{l}\text { Meat and edible meat } \\
\text { offal, n.e.s. }\end{array}$ & 5.23 & 2.43 & 3.98 & 3.41 & 3.39 & 4.47 & 6.06 & 5.20 & 5.40 & 5.30 & 5.71 & 5.65 & 5.36 \\
\hline 022 & Milk and cream & 3.72 & 4.45 & 2.61 & 2.51 & 2.25 & 2.07 & 1.98 & 1.93 & 1.45 & 2.47 & 2.03 & 2.16 & 1.67 \\
\hline 023 & Butte & 4.83 & (.) & 0 & 4. & 3. & 0.0 & 0.47 & -0.98 & -0. & -0.20 & 0.01 & 0.30 & 0.00 \\
\hline 024 & Cheese and curd & 2.35 & 2.33 & 1.5 & 2.37 & 2.83 & 2.29 & 2.43 & 1.27 & 1.14 & 0.89 & 1.30 & 0.75 & 0.90 \\
\hline 025 & Eggs fresh & 1.27 & 0.71 & 2.57 & 8.61 & 0.56 & 8.54 & 2.59 & 3.03 & 1.41 & 3.22 & 3.95 & 3.88 & 2.92 \\
\hline 034 & $\begin{array}{l}\text { Fish fresh, chilled or } \\
\text { frozen }\end{array}$ & 0.85 & 0.67 & 0.55 & 0.44 & 0.76 & 0.33 & -0.77 & -1.17 & -1.15 & -1.15 & -1.29 & -1.01 & -1.10 \\
\hline 035 & Fish dried, salted, smoked & -1.91 & -3.51 & -1.18 & 4.87 & 2.40 & 2.92 & 4.00 & 3.11 & 3.71 & 4.09 & 3.91 & 3.29 & 1.73 \\
\hline 036 & $\begin{array}{l}\text { Crustaceans and molluscs } \\
\text { (also in shell) }\end{array}$ & (.) & (.) & -4.11 & -5.66 & -5.18 & (.) & (.) & -9.43 & -3.64 & -8.14 & -12.9 & -8.30 & -7.41 \\
\hline 037 & $\begin{array}{l}\text { Fish, crustaceans and } \\
\text { molluscs prepared or } \\
\text { preserved }\end{array}$ & 0.17 & 0.71 & 4.19 & 5.85 & 3.54 & 3.60 & 3.00 & 3.30 & 2.79 & 3.02 & 1.85 & 3.82 & 2.78 \\
\hline
\end{tabular}




\begin{tabular}{|c|c|c|c|c|c|c|c|c|c|c|c|c|c|c|}
\hline 041 & Wheat & (.) & -2.06 & (.) & (.) & (.) & 0.42 & 1.84 & -5.96 & 2.96 & 4.57 & 4.25 & 5.44 & 8.26 \\
\hline 042 & Rice & -4.34 & -3.94 & -1.54 & -0.68 & 0.23 & -0.01 & -0.33 & -0.31 & 0.84 & -0.25 & 0.16 & -0.50 & -0.26 \\
\hline 043 & Barley, unmilled & (.) & (.) & -9.18 & -10.5 & (.) & (.) & (.) & (.) & (.) & (.) & (.) & (.) & -6.49 \\
\hline 044 & Maize & (.) & (.) & (.) & (.) & (.) & (.) & (.) & 8.61 & (.) & 8.25 & -1.39 & (.) & (.) \\
\hline 045 & Cereals, unmilled & (.) & (.) & (.) & (.) & 3.22 & (.) & (.) & -0.82 & 2.74 & 5.80 & -0.20 & (.) & 1.61 \\
\hline 046 & Meal and flour of wheat & -1.94 & 0.18 & 4.39 & 3.63 & 2.61 & 3.18 & 2.32 & 1.59 & 0.96 & 1.29 & 2.42 & 1.40 & 1.38 \\
\hline 047 & Cereal meals and flours & -3.06 & -2.62 & -1.58 & 0.08 & 1.01 & 2.96 & 3.51 & 3.98 & 1.46 & 2.00 & 3.10 & 2.60 & 2.53 \\
\hline 048 & $\begin{array}{l}\text { Cereal preparations and } \\
\text { preparations of flour }\end{array}$ & 0.92 & 1.53 & 1.30 & 1.73 & 1.26 & 1.06 & 1.23 & 0.86 & 0.50 & 1.02 & 1.17 & 1.28 & 1.54 \\
\hline 054 & $\begin{array}{l}\text { Vegetables fresh, chilled } \\
\text { or frozen }\end{array}$ & 3.48 & 3.26 & 2.80 & 3.05 & 3.31 & 3.28 & 2.29 & 1.43 & 2.51 & 2.49 & 1.80 & 2.03 & 2.09 \\
\hline 056 & $\begin{array}{l}\text { Vegetables root and } \\
\text { tubers, prepared }\end{array}$ & 2.46 & 0.85 & 1.10 & 2.11 & 1.88 & 1.72 & 2.06 & 1.66 & 2.19 & 2.33 & 2.67 & 2.20 & 1.54 \\
\hline 057 & $\begin{array}{l}\text { Fruit and nuts fresh or } \\
\text { dried }\end{array}$ & 2.77 & 2.79 & 2.72 & 1.72 & 0.41 & 0.58 & 0.25 & -0.10 & 0.34 & -0.02 & -0.12 & -0.22 & -0.19 \\
\hline 058 & $\begin{array}{l}\text { Fruit preserved and fruit } \\
\text { preparations }\end{array}$ & 2.64 & 1.68 & 2.11 & 2.33 & 1.69 & 1.19 & 1.49 & 1.32 & 1.27 & 1.37 & 1.18 & 1.12 & 1.19 \\
\hline 059 & $\begin{array}{l}\text { Fruit juices and vegetable } \\
\text { juices }\end{array}$ & 3.92 & 2.74 & 5.04 & 3.64 & 4.99 & 3.20 & 1.23 & 1.31 & 2.98 & 2.66 & 2.34 & 1.79 & 2.05 \\
\hline 061 & $\begin{array}{l}\text { Sugar, molasses and } \\
\text { honey }\end{array}$ & 3.12 & 2.21 & 1.53 & 1.63 & 0.03 & -0.42 & 0.24 & -1.07 & -0.27 & 0.37 & 0.17 & 0.33 & 0.29 \\
\hline 062 & Sugar confectionery & 0.54 & 0.83 & 1.06 & 1.15 & 1.49 & 1.62 & 1.55 & 1.01 & 1.01 & 0.85 & 0.73 & 1.13 & 0.65 \\
\hline 071 & $\begin{array}{l}\text { Coffee and coffee } \\
\text { substitutes }\end{array}$ & -2.43 & -2.61 & -1.74 & -1.20 & -1.18 & -1.30 & -1.45 & -1.51 & -1.66 & -1.37 & -0.79 & -0.33 & -0.40 \\
\hline 072 & Cocoa & -9.80 & -6.43 & -1.66 & -5.30 & -4.94 & -4.32 & -4.90 & -5.12 & 2.99 & -1.22 & -0.43 & 3.25 & -2.97 \\
\hline 073 & $\begin{array}{l}\text { Chocolate and other food } \\
\text { preparations containing } \\
\text { cocoa }\end{array}$ & 1.41 & 1.34 & 2.83 & 3.08 & 2.52 & 2.85 & 3.53 & 2.61 & 2.00 & 1.81 & 1.69 & 1.34 & 1.15 \\
\hline 074 & Tea & -1.74 & -0.51 & -0.59 & -0.68 & -1.01 & -2.77 & -2.25 & -1.75 & -0.39 & 0.72 & 1.19 & 0.13 & 1.12 \\
\hline 075 & Spices & -2.88 & -0.21 & -0.42 & 0.63 & 0.20 & 0.33 & 0.11 & 0.00 & -0.87 & -0.60 & 0.64 & 0.20 & 0.24 \\
\hline 081 & Feeding stuff for animals & 0.82 & 0.72 & -0.55 & -0.54 & -0.58 & -1.88 & 0.26 & -0.10 & -0.41 & -0.19 & -0.47 & -0.33 & -0.63 \\
\hline 091 & Margarine and shortening & -7.43 & -4.30 & -2.16 & 0.58 & -0.21 & -1.21 & -2.86 & -2.26 & -0.79 & -0.81 & -0.85 & -0.09 & 0.76 \\
\hline 098 & $\begin{array}{l}\text { Edible products and } \\
\text { preparations, n.e.s. }\end{array}$ & 0.65 & 0.68 & 0.24 & 0.24 & 0.48 & 0.66 & 0.61 & 0.56 & 0.54 & 0.64 & 0.49 & 0.25 & -0.04 \\
\hline 111 & Non-alcoholic beverages & 6.81 & 3.69 & 3.95 & 3.28 & 2.39 & 1.56 & 1.52 & 1.65 & 2.02 & 2.62 & 3.58 & 2.94 & 2.70 \\
\hline 112 & Alcoholic beverages & -0.43 & -0.93 & -0.40 & -0.83 & -1.21 & -1.93 & -2.43 & -3.82 & -3.41 & -3.14 & -2.44 & -2.22 & -2.12 \\
\hline 121 & $\begin{array}{l}\text { Tobacco unmanufactured; } \\
\text { tobacco refuse }\end{array}$ & -8.68 & -2.52 & 0.69 & 0.17 & -1.68 & -3.59 & -2.53 & -3.11 & -1.39 & -4.04 & -6.44 & -5.39 & -5.79 \\
\hline 122 & Tobacco manufactured & -5.36 & -0.34 & 1.52 & 2.99 & 1.06 & 1.49 & 4.19 & 7.15 & 1.03 & 0.80 & 1.14 & 0.97 & 0.71 \\
\hline 411 & Animal oils and fats & -5.01 & -3.53 & -1.95 & -0.33 & -0.60 & -1.57 & -1.58 & -1.37 & -1.43 & -0.66 & -1.05 & -0.67 & -1.05 \\
\hline 421 & $\begin{array}{l}\text { Fixed vegetable fats, and } \\
\text { oils "soft", crude, refined }\end{array}$ & -6.90 & -4.90 & -1.96 & -1.84 & -0.70 & 3.22 & 1.74 & -1.95 & -2.76 & -1.90 & -1.63 & -1.68 & -1.05 \\
\hline 422 & $\begin{array}{l}\text { Fixed vegetable fats, and } \\
\text { oils crude, refined other } \\
\text { than "soft" }\end{array}$ & -7.55 & -8.31 & -5.61 & -3.70 & -2.68 & -3.57 & -3.20 & -3.05 & -2.89 & -3.13 & -4.09 & -3.28 & -3.36 \\
\hline 431 & $\begin{array}{l}\text { Animal or vegetable fats } \\
\text { and oils processed }\end{array}$ & -8.72 & (.) & -6.71 & -5.12 & -4.74 & -4.38 & -3.87 & -3.47 & -3.94 & -1.72 & -3.76 & -4.70 & -4.29 \\
\hline
\end{tabular}

(.) - data not available.

Source: Authors' own calculations based on Eurostat, http://ec.europa.eu/eurostat/data/database. 
Table 3. The revealed comparative advantage indexes LFIi in trade in agri-food products between Poland and the United Kingdom in the years 2004-2016 according to the SITC classification

\begin{tabular}{|c|c|c|c|c|c|c|c|c|c|c|c|c|c|c|}
\hline SITC & Specification & 2004 & 2005 & 2006 & 2007 & 2008 & 2009 & 2010 & 2011 & 2012 & 2013 & 2014 & 2015 & 2016 \\
\hline 001 & ve animals & -0.24 & -0.17 & .12 & 22 & 0.00 & 01 & 0.10 & -0.40 & .15 & .23 & 0.41 & .45 & 0.48 \\
\hline 011 & sh, chi & (.) & 0.65 & 0.31 & 0.31 & 0.53 & 0.47 & 0.00 & 0.15 & 0.46 & 0.54 & 0.40 & 0.73 & 0.74 \\
\hline 012 & $\begin{array}{l}\text { Meat and edible meat } \\
\text { offal, fresh, chilled or } \\
\text { frozen (except meat of } \\
\text { bovine animals) }\end{array}$ & -0.10 & -1.06 & 0.60 & -4.01 & -7.21 & -5.93 & -6.26 & -3.76 & -4.45 & -4.02 & -3.17 & -1.96 & -1.19 \\
\hline 016 & $\begin{array}{l}\begin{array}{l}\text { offal salted, } \\
\text { smoked }\end{array}\end{array}$ & -0.01 & 0.01 & (.) & (.) & 2.86 & 3.40 & 3.04 & 2.87 & 2.03 & 2.15 & 2.14 & 1.62 & 1.45 \\
\hline 017 & $\begin{array}{l}1 \\
0\end{array}$ & 3.13 & 0.93 & 1.46 & 2.05 & 2.42 & 2.92 & 3.22 & 3.82 & 2.94 & 3.19 & 3.40 & 3.40 & 3.57 \\
\hline 022 & & 0.16 & 0.33 & 0.27 & 0.28 & 0.28 & 0.38 & 0.45 & 0.53 & 0.32 & 0.62 & 0.54 & 0.55 & 0.58 \\
\hline 023 & ttte & 0.06 & (.) & -0.07 & 0.10 & 0.07 & -0.02 & 0.00 & -0.23 & -0.10 & -0.10 & -0.06 & -0.02 & -0.08 \\
\hline 024 & & 0.15 & 0.43 & 0.09 & 0.34 & 0.76 & 0.58 & 0.53 & 0.50 & 0.36 & 0.28 & 0.43 & 0.12 & 0.22 \\
\hline 025 & Eggs & 0.01 & -0.01 & 0.07 & 0.13 & 0.00 & 0.11 & 0.08 & 0.11 & 0.20 & 0.12 & 0.14 & 0.13 & 0.13 \\
\hline 034 & (n) & -1.03 & -0.83 & .31 & -0.99 & 0.32 & 0.05 & -2.27 & -3.02 & -2.87 & -3.23 & -4.06 & -1.72 & -2.28 \\
\hline 035 & ed, salted, smoked & -0.01 & -0.01 & -0.01 & 0.10 & 0.05 & 0.31 & 0.31 & 0.19 & 0.19 & 0.19 & 0.24 & 0.22 & 0.09 \\
\hline 036 & & (.) & (.) & 0.00 & -0.08 & -0.08 & (.) & (.) & -0.02 & -0.04 & -0.08 & -0.12 & -0.05 & -0.12 \\
\hline 037 & Fish, & -0.02 & -0.01 & 0.79 & 1.37 & 1.39 & 1.66 & 1.49 & 1.29 & 1.03 & 0.86 & 0.74 & 0.76 & 0.75 \\
\hline 041 & $\mathrm{~V}$ & (.) & 0.00 & -0.10 & (.) & (.) & 0.00 & 0.02 & -0.37 & 0.50 & 0.23 & 0.22 & 0.10 & 0.06 \\
\hline 042 & Rice & -0.01 & -0.03 & -0.01 & -0.01 & 0.00 & 0.00 & -0.01 & 0.00 & 0.00 & -0.01 & -0.01 & -0.06 & -0.02 \\
\hline 043 & B & (.) & (.) & -0.35 & -0.07 & (.) & (.) & (.) & (.) & (.) & (.) & (.) & (.) & -0.02 \\
\hline 044 & . & (.) & (.) & (.) & (.) & (.) & (.) & (.) & 0.05 & (.) & 0.25 & -0.09 & (.) & (.) \\
\hline 045 & C & (.) & (.) & (.) & (.) & 0.01 & (.) & (.) & 0.00 & 0.00 & 0.00 & 0.00 & (.) & 0.00 \\
\hline 046 & at & -0.05 & -0.01 & 0.01 & 0.04 & 0.10 & 0.16 & 0.16 & 0.12 & 0.05 & 0.07 & 0.13 & 0.07 & 0.06 \\
\hline 047 & Cere & -0.12 & -0.14 & -0.10 & -0.04 & 0.02 & 0.03 & 0.05 & 0.06 & 0.02 & 0.05 & 0.04 & 0.05 & 0.05 \\
\hline 048 & C & -0.22 & 0.61 & 57 & 1.18 & 1.15 & 1.48 & 1.43 & 1.19 & 021 & 0.82 & 1.12 & 1.19 & 1.81 \\
\hline 054 & & 2.89 & 4.40 & 3.56 & 3.39 & 3.79 & 3.40 & 2.68 & 1.95 & 2.08 & 1.84 & 1.50 & 1.46 & 1.50 \\
\hline 056 & & 0.21 & -0.03 & 004 & 0.38 & 0.55 & 054 & 0.52 & 0.49 & 0.43 & 0.47 & 0.52 & 0.43 & 0.31 \\
\hline 057 & F & 0.42 & 0.57 & 0.68 & 0.44 & -0.10 & 0.20 & -0.11 & 0.21 & -0.01 & -0.35 & -0.51 & -0.69 & -0.49 \\
\hline 058 & & 1.02 & 0.66 & 080 & 0.72 & 0.80 & 0.57 & 0.54 & 0.59 & 0.53 & 0.46 & 0.39 & 0.30 & 0.32 \\
\hline 059 & F & 1.21 & 1.34 & 1.7 & 1.32 & 2.22 & 1.56 & 0.64 & 0.76 & 1.3 & .47 & 0.80 & 0.44 & 0.55 \\
\hline 061 & and & 0.70 & 0.35 & 0.20 & 0.17 & -0.14 & -0.58 & -0.08 & -0.61 & -0.36 & -0.03 & -0.09 & -0.05 & -0.05 \\
\hline 062 & & -0.26 & 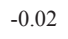 & 00 & & 0.23 & & 0.25 & 0.20 & 6 & 0.10 & 0.07 & 0.18 & 0.05 \\
\hline 071 & C & -3.19 & -3.50 & -2.84 & -2.57 & -2.29 & -1.91 & -2.34 & -2.40 & -3.20 & -2.73 & -1.27 & -0.64 & -0.65 \\
\hline 072 & Cocoa & $\mid-1.39$ & -0.57 & -0.13 & -0.80 & -0.83 & -0.60 & -0.66 & -0.99 & -0.21 & -0.03 & -0.01 & -0.30 & -0.18 \\
\hline 073 & parations containing & 0.58 & 0.95 & 2.57 & 1.79 & 2.67 & 3.92 & 5.98 & 6.86 & 4.97 & 4.11 & 3.37 & 2.60 & 2.04 \\
\hline 074 & Tea & -0.40 & -0.18 & -0.14 & -0.19 & -0.52 & -2.82 & -3.37 & -2.76 & -0.39 & 0.07 & 0.18 & -0.09 & 0.14 \\
\hline 075 & Spices & -0.02 & -0.01 & -0.03 & -0.01 & -0.02 & 0.00 & -0.01 & -0.01 & -0.11 & -0.10 & 0.01 & -0.02 & -0.02 \\
\hline 081 & Feedino & -0.92 & -0.47 & -4.41 & -1.98 & -1.48 & -6.77 & -0.13 & -0.20 & -0.49 & -0.61 & -1.08 & -0.86 & -1.13 \\
\hline 091 & Margarine and shortening & -0.29 & -0.06 & -0.09 & 0.00 & -0.05 & -0.33 & -0.89 & -1.34 & -0.37 & -0.34 & -0.39 & -0.25 & 0.06 \\
\hline
\end{tabular}




\begin{tabular}{|c|c|c|c|c|c|c|c|c|c|c|c|c|c|c|}
\hline 098 & $\begin{array}{l}\text { Edible products and } \\
\text { preparations, n.e.s. }\end{array}$ & -1.47 & -0.72 & -1.66 & -1.56 & -0.30 & 0.86 & 0.46 & 0.88 & 0.39 & 0.38 & 0.04 & -0.71 & -1.27 \\
\hline 111 & Non-alcoholic beverages & 1.26 & 1.16 & 1.19 & 0.68 & 0.87 & 0.65 & 0.46 & 0.48 & 0.45 & 0.56 & 0.62 & 0.57 & 0.57 \\
\hline 112 & Alcoholic beverages & -1.95 & -3.49 & -3.05 & -4.21 & -4.37 & -3.98 & -4.87 & -5.68 & -5.69 & -6.48 & -4.56 & -4.69 & -5.00 \\
\hline 21 & $\begin{array}{l}\text { Tobacco unmanufactured; } \\
\text { tobacco refuse }\end{array}$ & -0.14 & -0.12 & -0.01 & -0.01 & -1.34 & -1.16 & -1.46 & -1.14 & -0.05 & -0.34 & -0.62 & -1.99 & -1.87 \\
\hline 122 & Tobacco manufactured & -0.09 & -0.03 & 0.20 & 0.69 & 0.27 & 0.83 & 0.70 & 0.82 & 0.31 & 0.21 & 0.42 & 0.48 & 0.34 \\
\hline 11 & Animal oils and fats & -0.44 & -0.38 & -0.25 & -0.11 & -0.12 & -0.25 & -0.17 & -0.13 & -0.19 & -0.07 & -0.09 & -0.06 & -0.10 \\
\hline 21 & $\begin{array}{l}\text { Fixed vegetable fats, and } \\
\text { oils "soft", crude, refined }\end{array}$ & -0.04 & -0.08 & -0.02 & -0.01 & -0.05 & 0.56 & 0.18 & -0.44 & -0.69 & -0.12 & -0.53 & -0.55 & -0.24 \\
\hline 22 & $\begin{array}{l}\text { Fixed vegetable fats, and } \\
\text { oils crude, refined other } \\
\text { than "soft" }\end{array}$ & -0.26 & -0.24 & -0.16 & -0.09 & -0.12 & -0.15 & -0.18 & -0.09 & -0.10 & -0.07 & -0.25 & -0.12 & -0.16 \\
\hline 431 & $\begin{array}{l}\text { Animal or vegetable fats } \\
\text { and oils processed }\end{array}$ & -0.44 & (.) & -0.40 & -0.15 & -0.14 & -0.08 & -0.12 & -0.08 & -0.12 & -0.11 & -0.13 & -0.13 & -0.12 \\
\hline
\end{tabular}

(.) - data not available.

Source: Authors' own calculations based on Eurostat, http://ec.europa.eu/eurostat/data/database.

By analysing $R C A_{i}$ values set for trade with the United Kingdom (see Table 2), it can be seen that in the period considered Poland had a relative advantage for most agri-food products. A particularly high surplus was observed in the case of trade in less processed goods, e.g., meat and edible offal $\left(R C A_{016}=6.27, R C A_{017}=5.36\right.$, in 2016) and wheat $\left(R C A_{041}=8.26\right)$.

Good results obtained in this area were a consequence of the price advantage of Polish meat and grain producers. These advantages were achievable mainly due to Poland's lower labour costs and prices of land, as compared with those in the UK. According to a study carried out by Judzińska (2014), the production of poultry meat was characterized by high price competitiveness, especially after 2013 . At that time, the price of poultry meat on the UK market increased by $8 \%$, which allowed Polish producers to gain a very competitive position in the market.

Poland also had significant advantages in non-alcoholic beverages $\left(R C A_{111}=2.70\right.$, in $2016)$ and fruit juices and vegetable juices $\left(R C A_{059}=2.05\right)$. In this segment, price advantages were of key importance. According to studies conducted by Kozień (2014), investments made in the non-alcoholic beverage and fruit and vegetable processing industries contributed significantly to the increase in productivity and the attractiveness of selections to customers, not only on the domestic market but also on foreign ones, including the UK market.

A relatively favourable situation also occurred in the field of dairy products, especially in the milk and cream commodity group $\left(R C A_{022}=1,67\right.$, in 2016) and the eggs group $\left(R C A_{025}=2,92\right.$, in 2016). Slight relative advantages $\left(R C A_{i}<1\right)$ were found for the following products: cheese and curd, sugar, molasses and honey, spices, margarine and shortening, manufactured tobacco.

Lack of comparative advantages occurred for all goods included in the SITC 4 section - animal or vegetable oils, fats and waxes, and alcoholic beverages. Also, Poland did not have any comparative advantage in live animals, crustaceans and molluscs prepared or preserved, coffee and cocoa.

Analysis of the $L F I_{i}$ index that was used to examine the competitiveness of individual commodity groups in Polish-British trade, in the context of bilateral trade in merely agrifood products, in principle, confirms the earlier conclusions. Poland's comparative advantages in this bilateral trade emerged in almost the same product groups. In some cases, however, there were some differences with regard to their level. This is completely 
justified due to the specific design of the $L F I_{i}$ index. In Poland, the SITC 017 - meat and edible meat offal, n.e.s. $\left(L F I_{017}=3.57\right.$, in 2016) had a very high level of comparative advantages, when compared against the results of the agri-food sector. A slightly higher index level was also noted for trade in chocolate and other food preparations containing cocoa and cereal preparations and preparations of flour (see Table 3).

Analysis of the comparative advantages in agri-food products trade shows that in the years 2004-2016, trade in products from the SITC 4 section - animal or vegetable oils, fats and waxes exhibited the most advantageous situation among all agri-food products, and in particular SITC 431 - animal or vegetable fats and processed oils and SITC 422 - fixed vegetable fats and oils, refined other than 'soft'. Unfavourable $L F I_{i}$ levels could be found in trade in tobacco, alcohols and live animals.

\section{Conclusions}

The presented results of the analysis showed that during its period of EU membership, Poland's competitive position in trade in agri-food products with the United Kingdom improved. This is evidenced by the dynamically growing trade volume as well as by the positive trade balance that continues to increase from year to year. An improvement in the competitive position is also confirmed by the analysis made with the use of the comparative advantage indexes.

Particularly high levels of indicators were achieved in the meat and cereal-grain products and non-alcoholic beverages categories.

As follows from a number of studies (see, for instance Ambroziak, 2013; Pietrzak, Łapińska, 2015; Szczepaniak, 2014) the increase in the competitiveness of Polish products on the EU market, including the UK market, was mainly due to the price advantage of Polish food producers. Also, qualitative advantages related to the use of high quality raw materials in production and modern manufacturing technologies contributed significantly.

The analyses conducted also show that in some product groups Poland did not have, or had lost, its previously possessed comparative advantages (see, for instance, SITC 034 fish fresh, chilled or frozen, SITC 057 - fruit and nuts fresh or dried) in trade with the UK. However, it is worth emphasizing that the share of trade in products in which the comparative advantage ratios were negative was decreasing systematically. This, in turn, translated into an improvement in the balance of agricultural and food products in the Polish-British trade.

The increase in the competitiveness of Polish products on the British market was primarily due to the price advantage of Polish food producers. Noteworthy were the qualitative advantages associated with the use of high quality raw materials for production and modern manufacturing technologies.

The United Kingdom is a country, which, after EU enlargement, has become the most important migration destination for citizens of many countries. In this context, it is worth pointing out that the significant growth of Polish agri-food exports to the United Kingdom is also related to this fact. After Poland's accession to the European Union, Polish citizens were very willing to migrate to this country. Many of them set up their own companies to import Polish goods to the British market. Polish products through their presence on the British market gained new loyal buyers and these buyers were not only of Polish origin. 
In 2016, in a referendum on the United Kingdom's membership in the European Union, the British voted in favour of leaving the Community. For the time being it is not clear when this will happen and how the whole procedure will be carried out.

Certainly, significant changes should be expected in terms of migration and social policies. They will also affect Polish citizens. In the most pessimistic scenario, people who have lived only a short time in the UK and do not have a permanent residence permit may be asked to leave the country. In truth, this will likely depend on how much they will be needed on the UK labour market. In this context, it is worth pointing out that Polish citizens make up an important group of buyers on the British market that buy Polish food products. Thus, they contribute significantly to the achievement of favourable results in food products trade with the UK.

Difficulties may also be encountered by people who have established their businesses in the UK. Poles were eager to set up businesses in the UK because of a friendly tax system. Such benefits may also be lost. In the context of foreign trade, it is worth remembering that Polish companies provide services on the British market, selling Polish products, including agricultural and food products. At the same time, they promote brands that are highly respected and trusted by customers in Poland.

Leaving the EU also means a change in the rules that will apply to trade between the UK and the EU. It is difficult to assess now the possible effects of the return of restrictions on the flow of goods and capital. Great Britain will most likely seek to sign a new economic cooperation agreement with the EU. However, it cannot be ruled out that a possible consensus will not be reached.

The economic relations between the United Kingdom and EU countries, including Poland, are quite strong. As a result, both sides have a lot to lose. However, it seems that the situation of Poland is more difficult. The huge surplus in foreign trade makes Poland more dependent on exports to the United Kingdom than on imports from that country. By the asymmetry of export indexes, Poland's bargaining position in negotiations with the United Kingdom is going to be much weaker.

Agri-food products comprise an important group of exported products. If the United Kingdom leaves the common market and the customs union, then its trade with the EU will follow the WTO rules. The agri-food sector is likely to lose a lot (Rosati, 2016). It cannot be ruled out that the current extremely favourable situation for Poland in the foreign trade in agri-food products with the United Kingdom may change.

\section{References}

Ambroziak, Ł. (2013). Konkurencyjność eksportu rolno-spożywczego Polski i wybranych nowych państw członkowskich na rynku UE-15. Roczniki Naukowe Stowarzyszenia Ekonomistów Rolnictwa i Agrobiznesu, 15(3), 21-26.

Ambroziak, Ł. (2016). Konkurencyjność cenowo-jakościowa polskich producentów żywności na rynku niemieckim. Zeszyty Naukowe Szkoły Głównej Gospodarstwa Wiejskiego Problemy Rolnictwa Światowego, 16(1), 7-24.

Balassa, B. (1965). Trade Liberalization and "Revealed" Comparative Advantage. The Manchester School, 33, 99-123.

Bugamelli, M. (2001). Il modello di specializzazione internazionale dell'area dell'euro e dei principali paesi europei: omogeneità e convergenza. Banca d'Italia, Temi di discussione del Servizio Studi n. 402. Accessed from: http://www.bancaditalia.it/pubblicazioni/econo/temidi/td01/td402_01/td402/tema_402_01.pdf. 
Firlej, K. (2010). Ocena konkurencyjności i szans rozwoju przedsiębiorstw przemysłu rolno-spożywczego w warunkach unijnych. Roczniki Ekonomiczne Kujawsko-Pomorskiej Szkoły Wyższej w Bydgoszczy, 3, $163-175$.

Eurostat. Accessed from: http://ec.europa.eu/eurostat/data/database.

Gehrke, B., Grupp, H. (1994). Innovationspotential und Hochtechnologie, Physica-Verlag.

Hoen, A. R., Oosterhaven, J. (2006). On the Measurement of Comparative Advantage. The Annals of Regional Science, 40(3), 677-691.

Judzińska, A. (2014). Konkurencyjność cenowa polskiego sektora mięsnego w Unii Europejskiej. Roczniki Naukowe Stowarzyszenia Ekonomistów Rolnictwa i Agrobiznesu, 16(6), 189-195.

Kozień, E. (2014). Perspektywy rozwoju branży owocowo-warzywnej, in: K. Firlej (ed.), Kierunki i perspektywy rozwoju wybranych branż przemysłu rolno-spożywczego w Polsce, Uniwersytet Ekonomiczny w Krakowie, Kraków.

Lafay, G. (1992). The Measurement of Revealed Comparative Advantages, in: Dagenais, M. G., Mue,t P.-A., (eds.), International Trade Modelling, Chapman\&Hall, London.

Łapińska, J. (2014). Determinants of intra-industry trade in agricultural and food products between Poland and EU countries. Danube, 5(3), 159-172.

Misala, J. (2007). Międzynarodowa zdolność konkurencyjna i międzynarodowa konkurencyjność gospodarki narodowej. Podstawy teoretyczne, Wydawnictwo Politechniki Radomskiej, Radom.

Pawlak, K. (2014). Zmiany w polskim handlu zagranicznym produktami rolno-spożywczymi po akcesji do Unii Europejskiej. Zeszyty Naukowe Szkoły Głównej Gospodarstwa Wiejskiego Problemy Rolnictwa Światowego, 14(2), 123-131.

Pietrzak, M. B., Łapińska, J. (2015). Determinants European Union's Trade - Evidence from a Panel Estimation of the Gravity Model, E\& M Ekonomie a Management, 18(1), 18-27.

Rocznik Statystyczny Handlu Zagranicznego 2016, (2016). Główny Urząd Statystyczny, Warszawa.

Rosati, D. K. (2016). Poland-UK relations after Brexit: Suggested priorities for negotiations, in: Ch. Wyplosz, What To Do With the UK? EU perspectives on Brexit, AVoxEU.org Book, CEPR Press, London, 99-106.

Szczepaniak, I. (2011). Konkurencyjność polskiego sektora rolno-spożywczego na rynku nowych państw członkowskich Unii Europejskiej (UE-12). Zeszyty Naukowe Szkoły Głównej Gospodarstwa Wiejskiego Problemy Rolnictwa Światowego, 11(2), 125-137.

Szczepaniak I. (2014). Strategie konkurencji stosowane przez polskich producentów żywności na rynku Unii Europejskiej, in: A. Olszańska, J. Szymańska (eds.), Agrobiznes 2014. Rozwój agrobiznesu w okresie 10 lat przynależności Polski do Unii Europejskiej, Wydawnictwo Naukowe Uniwersytetu Ekonomicznego we Wrocławiu, Wrocław.

Szczepaniak, I. (ed.) (2015). Konkurencyjność polskich producentów żywności i jej determinanty, Instytut Ekonomiki Rolnictwa i Gospodarki Żywnościowej - Państwowy Instytut Badawczy, Warszawa.

Vollrath, T.L. (1991). A Theoretical Evaluation of Alternative Trade Intensity Measures of Revealed Comparative Advantage. Weltwirtschaftliches Archiv, 127(2), 265-280. 